\title{
UNIFORM POINTWISE ASYMPTOTICS OF SOLUTIONS TO QUASI-GEOSTROPHIC EQUATION
}

\author{
TOMASZ JAKUBOWSKI AND GRZEGORZ SERAFIN
}

\begin{abstract}
We provide two-sided pointwise estimates and uniform asymptotics of the solutions to the subcritical quasi-geostrophic equation with initial data in $L^{2 /(\alpha-1)}\left(\mathbb{R}^{2}\right)$. Furthermore, we give upper bound of similar type for any derivative of the solutions. Initial data in $L^{p}\left(\mathbb{R}^{2}\right)$, $p>2 /(\alpha-1)$, are also discussed.
\end{abstract}

\section{INTRODUCTION}

In this paper we study the two-dimensional dissipative quasi-geostrophic equation

$$
\left\{\begin{array}{l}
\theta_{t}+R^{\perp} \cdot \nabla \theta+(-\Delta)^{\alpha / 2} \theta=0 \\
\theta(0, x)=\theta_{0}(x)
\end{array}\right.
$$

Here, $R^{\perp}=\left(-R_{2}, R_{1}\right)$, where $R=\left(R_{1}, R_{2}\right)$ is the two-dimensional Riesz transform given by $R_{i}=\frac{\partial}{\partial x_{i}}(-\Delta)^{-1 / 2} \theta, i \in\{1,2\}$. Throughout the paper we assume $\alpha \in(1,2)$ and $\theta$ is a mild solution to the initial value problem (11), that is $\theta$ satisfies the following equation,

$$
\theta(t, \cdot)(x)=P_{t} \theta_{0}(x)+\int_{0}^{t} \int_{\mathbb{R}^{2}} \nabla p(t-s, x-y) \cdot R^{\perp} \theta(s, y) \theta(s, y) d y d s .
$$

For $\alpha=1$, the two-dimensional quasi-geostrophic equation is the analogue of the 3D NavierStokes equation and solutions to both equations admit similar behaviour [6]. The case $\alpha=1$ is therefore called critical exponent, while $\alpha \in(1,2)$ are subcritical exponents.

Solutions to the two-dimensional dissipative quasi-geostrophic equation model several phenomena (see [5, 20] ) and have been intensively studied for more than the last two decades. In 1995, Resnick [22] proved existence of strong solutions for $\theta_{0} \in L^{2}\left(\mathbb{R}^{2}\right)$ as well as the maximum principle

$$
\|\theta(t, \cdot)\|_{L^{p}} \leq\left\|\theta_{0}\right\|_{L^{p}}
$$

where $t \geqslant 0$ and $1<p \leq \infty$. This inequality has been improved in several directions by deriving a precise decay rate of $\|\theta(t, \cdot)\|_{L^{p}}$, see e.g. [7, 8, 4, 14, 18, 19]. In [4] authors considered the initial condition $\theta_{0} \in L^{p}$ with $p \geqslant \frac{2}{\alpha-1}$ and obtained many interesting bounds for $L^{q}, q \geqslant p$, norms of mild solutions to (1). In particular, they showed that for $\theta_{0} \in L^{\frac{2}{\alpha-1}}$ and any multi-index $\mathbf{k} \in \mathbb{N}^{2}$

$$
\lim _{t \rightarrow \infty} t^{\frac{|\mathbf{k}|}{\alpha}+\frac{d}{\alpha}\left(\frac{\alpha-1}{2}-\frac{1}{q}\right)}\left\|\nabla^{\mathbf{k}} \theta(t, \cdot)\right\|_{q}=0 .
$$

Under additional assumption $\theta_{0} \in L^{1}$, for every $\beta \in\left[0, \frac{1}{\alpha}\right)$ there is $C>0$ such that

$$
\left\|\nabla^{\mathbf{k}} \theta(t, \cdot)-\nabla^{\mathbf{k}}\left(P_{t} \theta_{0}\right)\right\|_{q} \leq C t^{-\frac{|k|}{\alpha}-\frac{d}{\alpha}\left(1-\frac{1}{q}\right)-\beta},
$$

where $\left(P_{t}\right)_{t \geqslant 0}$ is the semi-group generated by $\Delta^{\alpha / 2}$.

Although all of the aforementioned results provide precise bounds for $L^{p}$ norms of the solutions, they do not say much about pointwise behaviour of these solutions. In particular, there are not known any lower bounds. In fact, this is rather common problem in the theory of nonlinear

2010 Mathematics Subject Classification. 35B40; 35K55; 35S10.

Key words and phrases. Fractional Laplacian, Quasi-geostrophic equation, Pointwise estimates.

The paper is partially supported by the NCN grant 2015/18/E/ST1/00239. 
differential equations. Nevertheless, in this paper, we solve it for the dissipative quasi-geostrophic equation with nonnegative $\theta_{0} \in L^{\frac{2}{\alpha-1}}$ by giving two-sided pointwise estimates as well as some uniform asymptotics of mild solutions. The main results of the paper are stated in the following theorems.

Theorem 1.1. Let $\theta_{0} \in L^{\frac{2}{\alpha-1}}\left(\mathbb{R}^{2}\right)$ be nonnegative. There is a constant $C=C\left(\theta_{0}, \alpha\right)>1$ such that

$$
\frac{1}{C} P_{t} \theta_{0}(x) \leq \theta(t, x) \leq C P_{t} \theta_{0}(x)
$$

If we remove the nonnegativity condition, the upper bound $\theta(t, x) \leq C P_{t}\left|\theta_{0}\right|$ holds (see Theorem 1.3). Note that the semi-group $P_{t}$ and its kernel $p(t, x)$ are well known objects (see Section 2.1 for the details).

Theorem 1.2. For nonnegative $\theta_{0} \in L^{\frac{2}{\alpha-1}}\left(\mathbb{R}^{2}\right)$, we have

$$
\lim _{t \rightarrow 0}\left\|\frac{\theta(t, \cdot)}{P_{t} \theta_{0}}-1\right\|_{\infty}=\lim _{t \rightarrow \infty}\left\|\frac{\theta(t, \cdot)}{P_{t} \theta_{0}}-1\right\|_{\infty}=\lim _{|x| \rightarrow \infty} \sup _{t>0}\left|\frac{\theta(t, \cdot)}{P_{t} \theta_{0}}-1\right|=0 .
$$

Finally, we complete these results by establishing upper bounds for derivatives of the solutions:

Theorem 1.3. For $\theta_{0} \in L^{\frac{2}{\alpha-1}}$ and any multi-index $\mathbf{k} \in \mathbb{N} \times \mathbb{N}$, there is $C=C\left(\theta_{0}, \mathbf{k}, \alpha\right)>0$ such that

$$
\left|\nabla^{\mathbf{k}} \theta(t, x)\right| \leq C t^{-|\mathbf{k}| / \alpha} P_{t}\left|\theta_{0}\right|(x)
$$

Note that $\nabla^{\mathbf{k}} P_{t} \theta_{0}$ admits the same estimate (see (9)). It turns out that the power $p=\frac{2}{\alpha-1}$ in the initial condition $\theta_{0} \in L^{p}$ is critical in some sense. One could observe this phenomenon already in the paper [4]. Depending on $p$ is greater or less than $\frac{2}{\alpha-1}$, different difficulties occur and different behaviour of solutions is expected. Similar situation appears in the fractal Burgers equation, which has been studied by the authors in [12, 11] in case of (not only) critical power of the nonlinear drift term. The methods developed there have been improved and adapted to the quasi-geostrophic equation. Nevertheless, some ideas come from theory of linear perturbations of fractional Laplacian (see e.g. [3, 13]). In fact, the upper bound in (3) is concluded from [16], where also linear equations have been considered.

The paper is organized as follows. In Section 2 we gather some properties of the heat kernel $p(t, x)$ for $\Delta^{\alpha / 2}$ as well as some basic facts and initial results for Riesz transform. Section 3 is devoted to estimates and asymptotics of solutions to (1), while in Section 4 we prove the bound for theirs derivatives.

Throughout the paper, we write $f \approx g$ ( $f \lesssim g$ respectively) for $f, g \geq 0$, if there is a constant $c=c\left(\alpha, \theta_{0}\right) \geq 1$ such that $c^{-1} f \leq g \leq c f(f \leq c g$ respectively $)$ on their common domain. The constants $c, C, c_{i}$, whose exact values are unimportant, may be changed in each statement and proof. As usual, we write $a \wedge b:=\min (a, b)$ and $a \vee b:=\max (a, b)$.

\section{Preliminaries}

2.1. Stable semigroup. Throughout the paper we consider $\alpha \in(1,2)$. In this section we recall some well known results on the stable semigroup. Let

$$
\nu(z)=\frac{\alpha 2^{\alpha-1} \Gamma\left(1+\frac{\alpha}{2}\right)}{\pi \Gamma\left(1-\frac{\alpha}{2}\right)}|z|^{-2-\alpha}, \quad z \in \mathbb{R}^{2} .
$$

For (smooth and compactly supported) test function $\varphi \in C_{c}^{\infty}\left(\mathbb{R}^{2}\right)$, we define the fractional Laplacian by

$$
\Delta^{\alpha / 2} \varphi(x):=-(-\Delta)^{\alpha / 2} \varphi(x)=\lim _{\varepsilon \downarrow 0} \int_{\{|z|>\varepsilon\}}[\varphi(x+z)-\varphi(x)] \nu(z) d z, \quad x \in \mathbb{R}^{2}
$$


In terms of the Fourier transform, $\widehat{\Delta^{\alpha / 2} \varphi}(\xi)=-|\xi|^{\alpha} \widehat{\varphi}(\xi)$. Denote by $p(t, x-y)$ the fundamental solution of the equation $\partial_{t} u=\Delta^{\alpha / 2} u$, that is, for fixed $y \in \mathbb{R}^{2}, u(t, x)=p(t, x-y)$ solves

$$
\begin{cases}\partial_{t} u=\Delta^{\alpha / 2} u, & t>0, x \in \mathbb{R}^{2}, \\ u(0, x)=\delta_{y}(x), & x \in \mathbb{R}^{2} .\end{cases}
$$

It is well known that $p(\cdot, \cdot) \in C^{\infty}\left((0, \infty) \times \mathbb{R}^{2}\right)$ and $p(t, x)=p(t,-x)$ for any $t>0$ and $x \in \mathbb{R}^{2}$. It also enjoys the following scaling and semi-group properties

$$
\begin{aligned}
& p(t, x)=t^{-2 / \alpha} p\left(1, t^{-1 / \alpha} x\right), \quad t>0, x \in \mathbb{R}^{2}, \\
& p(t, x)=\int_{\mathbb{R}^{2}} p(t-s, x-z) p(s, z) d z, \quad t>s>0, x \in \mathbb{R}^{2},
\end{aligned}
$$

and pointwise estimates,

$$
p(t, x) \approx \frac{t}{\left(t^{1 / \alpha}+|x|\right)^{2+\alpha}} \approx t^{-2 / \alpha} \wedge \frac{t}{|x|^{2+\alpha}}, \quad t>0, x, y \in \mathbb{R}^{2} .
$$

For any multindex $\mathbf{k}=\left(k_{1}, k_{2}\right) \in \mathbb{N}^{2}$ we denote

$$
\nabla^{\mathbf{k}} f(x)=\frac{\partial^{|\mathbf{k}|}}{\partial x_{1}^{k_{1}} \partial x_{2}^{k_{2}}} f(x), \quad x=\left(x_{1}, x_{2}\right) \in \mathbb{R}^{2},
$$

where $|\mathbf{k}|=k_{1}+k_{2}$. By scaling property and [23, Lemma 3.1] (see also [15, 10] for more general setting),

$$
\left|\nabla^{\mathbf{k}} p(t, x)\right| \leq c_{\mathbf{k}} t^{-\frac{|\mathbf{k}|}{\alpha}} p(t, x), \quad t>0, x, y \in \mathbb{R}^{2} .
$$

From (9) we easily get the $L^{p}$-estimates:

$$
\left\|\nabla^{\mathbf{k}} p(t, \cdot)\right\|_{p} \lesssim t^{-\frac{2}{\alpha}\left(1-\frac{1}{p}\right)-\frac{|\mathbf{k}|}{\alpha}}
$$

We denote by $P_{t}$ the stable semigroup operator,

$$
P_{t} f(x)=\int_{\mathbb{R}^{2}} p(t, x-y) f(y) d y, \quad t>0, x \in \mathbb{R}^{2} .
$$

The name 'stable' comes from an $\alpha$-stable process, which is generated by $\Delta^{\alpha / 2}$ and the semigroup describes transition of probabilities (see, e.g. [1, 2]). For $f \in L^{\frac{2}{\alpha-1}}\left(\mathbb{R}^{2}\right)$ and $p \in\left[\frac{2}{\alpha-1}, \infty\right]$, the following estimate holds $([24])$,

$$
\left\|P_{t} f\right\|_{p} \lesssim t^{-\frac{(\alpha-1)}{\alpha}+\frac{2}{\alpha p}}\|f\|_{\frac{2}{\alpha-1}}
$$

In the lemma below, we note some additional decay properties.

Lemma 2.1. For $f \in L^{\frac{2}{\alpha-1}}\left(\mathbb{R}^{2}\right)$, we have

$$
\begin{array}{r}
\lim _{t \rightarrow 0}\left\|t^{\frac{\alpha-1}{\alpha}} P_{t} f\right\|_{\infty}=0, \\
\lim _{t \rightarrow \infty}\left\|t^{\frac{\alpha-1}{\alpha}} P_{t} f\right\|_{\infty}=0, \\
\lim _{|x| \rightarrow \infty} \sup _{t>0}\left|t^{\frac{\alpha-1}{\alpha}} P_{t} f(x)\right|=0 .
\end{array}
$$

Proof. The limit (12) follows from [4, (2.2)]. Next, for every $\varepsilon>0$ there is $R>0$ such that $\left\|f \mathbb{1}_{B(0, R)^{c}}\right\|_{\frac{2}{\alpha-1}}<\varepsilon$. By Young inequality and (9),

$$
\left\|P_{t}\left(f \mathbb{1}_{B(0, R)^{c}}\right)\right\|_{\infty} \leq\|p(t, \cdot)\|_{\frac{2}{3-\alpha}}\left\|f \mathbb{1}_{B(0, R)^{c}}\right\|_{\frac{2}{\alpha-1}} \leq c_{1} t^{\frac{1-\alpha}{\alpha}} \varepsilon
$$


Hence,

$$
\begin{aligned}
\left\|t^{\frac{\alpha-1}{\alpha}} P_{t} f\right\|_{\infty} & \leq t^{\frac{\alpha-1}{\alpha}}\left(\left\|P_{t}\left(f \mathbb{1}_{B(0, R)}\right)\right\|_{\infty}+\left\|P_{t}\left(f \mathbb{1}_{B(0, R)^{c}}\right)\right\|_{\infty}\right) \\
& \leq c_{1} t^{\frac{\alpha-1}{\alpha}}\left(\left\|p(t, \cdot)_{\infty}\right\|\left\|\mathbb{1}_{B(0, R)}\right\|_{\frac{2}{3-\alpha}}\|f\|_{\frac{2}{\alpha-1}}+t^{\frac{1-\alpha}{\alpha}} \varepsilon\right) \\
& \leq c_{2}\left(t^{\frac{\alpha-3}{\alpha}}+\varepsilon\right),
\end{aligned}
$$

which yields (13). Finally, for $|x|>2 R$ and $|y|<R$, by (86), we have $p(t, x-y) \lesssim t^{\frac{1-\alpha}{\alpha}}|x-y|^{\alpha-3} \lesssim$ $t^{\frac{1-\alpha}{\alpha}}|x|^{\alpha-3}$. Therefore, for $|x|>2 R$,

$$
\begin{aligned}
\sup _{t>0}\left|t^{\frac{\alpha-1}{\alpha}} P_{t} f(x)\right| & \leq \sup _{t>0} t^{\frac{\alpha-1}{\alpha}}\left(\left|P_{t}\left(f \mathbb{1}_{B(0, R)}\right)(x)\right|+\left\|P_{t}\left(f \mathbb{1}_{B(0, R)^{c}}\right)\right\|_{\infty}\right) \\
& \lesssim|x|^{\alpha-3}\left\|f \mathbb{1}_{B(0, R)}\right\|_{1}+\varepsilon \lesssim|x|^{\alpha-3}\|f\|_{\frac{2}{\alpha-1}}+\varepsilon
\end{aligned}
$$

and (14) holds.

2.2. Riesz transform. Let $R_{i}, i \in\{1,2\}$ be the Riesz transforms, i.e.

$$
R_{i} f(x)=c P . V . \int_{\mathbb{R}^{2}} \frac{y_{i}}{|y|^{3}} f(x-y) d y, \quad y=\left(y_{1}, y_{2}\right) \in \mathbb{R}^{2},
$$

where $c$ is some constant and P.V. denotes the principal value of the integral. Let $R=\left(R_{1}, R_{2}\right)$ and $R^{\perp}=\left(-R_{2}, R_{1}\right)$. It is clear that $\left|R^{\perp} f\right|=|R f|$.

It is well known that the Riesz transform is continuous on $L^{p}$ for $p \in(1, \infty)$, i.e. for $f \in L^{p}$ we have (see e.g. [9, Corollary 4.8])

$$
\|R f\|_{p} \leq c_{p}\|f\|_{p} .
$$

Proposition 2.2. For any multi-index $\mathbf{k} \in \mathbb{N} \times \mathbb{N}$ there is a constant $C>0$ such that

$$
\left|R^{\perp} \nabla^{\mathbf{k}} p(t, x)\right| \leq C t^{-\frac{|\mathbf{k}|}{\alpha}} \frac{1}{\left(t^{1 / \alpha}+|x|\right)^{2}}, \quad t>0, x \in \mathbb{R}^{2} .
$$

Proof. It is easy to see that both sides of (16) admit the scalling property $f(t, x)=t^{-(2+|\mathbf{k}|) / \alpha} f\left(1, t^{-1 / \alpha} x\right)$. Hence, it is enough to consider $t=1$. First, let us write

$$
\begin{aligned}
\left|R_{i} \nabla^{\mathbf{k}} p(1, x)\right| & =c\left|P . V . \int_{\mathbb{R}^{2}} \frac{y_{i}}{|y|^{3}} \nabla^{\mathbf{k}} p(1, x-y) d y\right| \\
& \leq c\left|P . V . \int_{|y| \leq 1} \frac{y_{i}}{|y|^{3}} \nabla^{\mathbf{k}} p(1, x-y) d y\right|+c \int_{|y|>1} \frac{1}{|y|^{2}}\left|\nabla^{\mathbf{k}} p(1, x-y)\right| d y .
\end{aligned}
$$

It follows from (9) that

$$
\sup _{|w| \leq 1} \mid \nabla\left(\nabla^{\mathbf{k}} p(1, x+w) \mid \lesssim t^{-(|\mathbf{k}|+1) / \alpha} p(t, x) .\right.
$$

Hence, since

$$
P . V . \int_{|y| \leq 1} \frac{y_{i}}{|y|^{3}} \nabla^{\mathbf{k}} p(1, x) d y=0,
$$

the mean value theorem gives us

$$
\begin{aligned}
\left|P . V . \int_{|y| \leq 1} \frac{y_{i}}{|y|^{3}} \nabla^{\mathbf{k}} p(1, x-y) d y\right| & =\left|P . V . \int_{|y| \leq 1} \frac{y_{i}}{|y|^{3}}\left(\nabla^{\mathbf{k}} p(1, x-y)-\nabla^{\mathbf{k}} p(1, x)\right) d y\right| \\
& =\mid \int_{|y| \leq 1} \frac{y_{i}}{|y|^{3}} y \cdot \nabla\left(\nabla^{\mathbf{k}} p\left(1, x+w_{y}\right) d y \mid\right. \\
& \leq \int_{|y| \leq 1} \frac{|y|^{2}}{|y|^{3}} \sup _{|w| \leq 1} \mid \nabla\left(\nabla^{\mathbf{k}} p(1, x+w) \mid d y \lesssim p(1, x) \lesssim \frac{1}{x^{2}+1} .\right.
\end{aligned}
$$


Next,

$$
\int_{|y|>|x| \vee 1} \frac{1}{|y|^{2}}\left|\nabla^{\mathbf{k}} p(1, x-y) d y\right| \lesssim \int_{|y|>1 \vee|x|} \frac{1}{1+|x|^{2}} p(1, x-y) d y \leq \frac{1}{1+|x|^{2}}
$$

which gives (16) for $|x| \leq 1$. Finally, for $1<|y| \leq|x|$, we have $\frac{1+|y|^{2+\alpha}}{|y|^{2}} \leq 2|y|^{\alpha} \leq 2|x|^{\alpha} \leq$ $2 \frac{2+|x|^{2+\alpha}}{|x|^{2}}$, which yields $\frac{1}{|y|^{2}} \lesssim \frac{p(1, y)}{p(2, x)\left(1+|x|^{2}\right)}$. Thus,

$$
\int_{1<|y| \leq|x|} \frac{1}{|y|^{2}}\left|\nabla^{\mathbf{k}} p(1, x-y) d y\right| \lesssim \int_{\mathbb{R}^{d}} \frac{1}{1+|x|^{2}} \frac{p(1, y) p(1, x-y)}{p(2, x)} d y=\frac{1}{1+|x|^{2}}
$$

Proposition 2.3. For every $\mathbf{k} \in \mathbb{N}^{2}$ there is a constant $C_{\mathbf{k}}>0$ such that for all $t>0$,

$$
\left\|\nabla^{\mathbf{k}} R^{\perp} P_{t} \varphi\right\|_{\infty} \leq C_{\mathbf{k}} t^{-\frac{|\mathbf{k}|+\alpha-1}{\alpha}}\|\varphi\|_{\frac{2}{\alpha-1}}, \quad \varphi \in L^{\frac{2}{\alpha-1}}\left(\mathbb{R}^{2}\right) .
$$

\section{Furthermore}

$$
\lim _{t \rightarrow 0}\left\|t^{\frac{\alpha-1}{\alpha}} R^{\perp} P_{t} \varphi\right\|_{\infty}=\lim _{t \rightarrow \infty}\left\|t^{\frac{\alpha-1}{\alpha}} R^{\perp} P_{t} \varphi\right\|_{\infty}=\lim _{|x| \rightarrow \infty} \sup _{t>0}\left|t^{\frac{\alpha-1}{\alpha}} R^{\perp} P_{t} \varphi(x)\right|=0 .
$$

Proof. First, by (15) and (10), we have

$$
\begin{aligned}
\left|\nabla^{\mathbf{k}} R_{i} P_{t} \varphi(x)\right| & =\left|\nabla^{\mathbf{k}} \int_{\mathbb{R}^{2}} p(t, x-y) R_{i} \varphi(y) d y\right| \\
& \leq \int_{\mathbb{R}^{2}}\left|\nabla^{k} p(t, x-y) R_{i} \varphi(y)\right| d y \\
& \leq\left\|\nabla^{\mathbf{k}} p(t, \cdot)\right\|_{\frac{2}{3-\alpha}}\left\|R_{i} \varphi\right\|_{\frac{2}{\alpha-1}} \lesssim t^{-(|k|+\alpha-1) / \alpha}\|\varphi\|_{\frac{2}{\alpha-1}},
\end{aligned}
$$

which gives (17). Let us fix $\varepsilon>0$. There are $M_{\varepsilon}>0$ and $R_{\varepsilon}$ such that $\left\|\varphi \mathbb{1}_{\left\{|\varphi|>M_{\varepsilon}\right\}}\right\|_{\frac{2}{\alpha-1}} \leq \varepsilon$ and $\left\|\varphi \mathbb{1}_{B\left(0, R_{\varepsilon}\right)^{c}}\right\|_{\frac{2}{\alpha-1}} \leq \varepsilon$. Hence, by (15) and (10),

$$
\begin{aligned}
& \int_{|\varphi|>M_{\varepsilon}}\left|R_{i} p(t, x-y) \varphi(y)\right| d y \lesssim\|p(t, \cdot)\|_{\frac{2}{3-\alpha}}\left(\int_{|\varphi|>M_{\varepsilon}}|\varphi(y)|^{\frac{2}{\alpha-1}} d y\right)^{\frac{\alpha-1}{2}} \leq \varepsilon t^{-\frac{\alpha-1}{\alpha}}, \\
& \int_{|y|>R_{\varepsilon}}\left|R_{i} p(t, x-y) \varphi(y)\right| d y \lesssim\|p(t, \cdot)\|_{\frac{2}{3-\alpha}}\left(\int_{|y|>R_{\varepsilon}} \varphi(y)^{\frac{2}{\alpha-1}} d y\right)^{\frac{\alpha-1}{2}} \leq \varepsilon t^{-\frac{\alpha-1}{\alpha}} .
\end{aligned}
$$

Thus, by (18), (15) and (10),

$$
\begin{aligned}
\left|R_{i} P_{t} \varphi(x)\right| & =\left|\int_{\mathbb{R}^{2}} R_{i} p(t, x-y) \varphi(y) d y\right| \\
& \leq \sqrt{M_{\varepsilon}} \int_{|\varphi| \leq M_{\varepsilon}}\left|R_{i} p(t, x-y)\right| \sqrt{|\varphi(y)|} d y+\int_{|\varphi|>M_{\varepsilon}}\left|R_{i} p(t, x-y) \varphi(y)\right| d y \\
& \lesssim \sqrt{M_{\varepsilon}}\left\|R_{i} p(t, \cdot)\right\|_{\frac{4}{5-\alpha}}\|\varphi\|_{\frac{2}{\alpha-1}}+\varepsilon t^{-\frac{\alpha-1}{\alpha}} \\
& \lesssim \sqrt{M_{\varepsilon}} t^{-\frac{\alpha-1}{2 \alpha}}+\varepsilon t^{-\frac{\alpha-1}{\alpha}},
\end{aligned}
$$

and consequently $\left\|t^{\frac{\alpha-1}{\alpha}} R^{\perp} P_{t} \varphi\right\|_{\infty} \lesssim \sqrt{M_{\varepsilon}} t^{\frac{\alpha-1}{2 \alpha}}+\varepsilon$, which proves the first limit from the assertion. 
Next, by (10), (16), (18) and (19), we get

$$
\begin{aligned}
\left|R_{i} P_{t} \varphi\right| & =\left|\int_{\mathbb{R}^{2}} R_{i} p(t, x-y) \varphi(y) d y\right| \\
& \leq M_{\varepsilon} \int_{\substack{|\varphi| \leq M_{\varepsilon} \\
|y| \leq R_{\varepsilon}}}\left|R_{i} p(t, x-y)\right| d y+\int_{|\varphi|>M_{\varepsilon}}\left|R_{i} p(t, x-y) \varphi(y)\right| d y+\int_{|y|>R_{\varepsilon}}\left|R_{i} p(t, x-y) \varphi(y)\right| d y \\
& \lesssim M_{\varepsilon} R_{\varepsilon}^{2} t^{-2 / \alpha}+\varepsilon t^{-\frac{\alpha-1}{\alpha}},
\end{aligned}
$$

which implies $\lim _{t \rightarrow \infty}\left\|t^{\frac{\alpha-1}{\alpha}} R^{\perp} P_{t} \varphi\right\|_{\infty}=0$. By virtue of the previous two limits, it is enough to prove that for any $0<t_{1}<t_{2}<\infty$,

$$
\lim _{|x| \rightarrow \infty} \sup _{t \in\left(t_{1}, t_{2}\right)}\left|R^{\perp} P_{t} \varphi(x)\right|=0 .
$$

By (15), (16), (19) and Hölder inequality, we get for $|x|>R_{\varepsilon}$ and $t \in\left(t_{1}, t_{2}\right)$,

$$
\begin{aligned}
\left|R_{i} P_{t} \varphi(x)\right| & =\left|\int_{\mathbb{R}^{2}} R_{i} p(t, x-y) \varphi(y) d y\right| \\
& \lesssim \int_{|y|>R_{\varepsilon}}\left|R_{i} p(t, x-y) \varphi(y)\right| d y+\int_{|y| \leq R_{\varepsilon}} \frac{1}{\left(t_{1}^{1 / \alpha}+|x-y|\right)^{2}} \varphi(y) d y \\
& \lesssim \varepsilon t_{1}^{-\frac{\alpha-1}{\alpha}}+\frac{1}{\left(|x|-R_{\varepsilon}\right)^{2}}\left(\int \mathbb{1}_{\left\{|y| \leq R_{\varepsilon}\right\}} d y\right)^{\frac{3-\alpha}{2}}\|\varphi\|_{\frac{2}{\alpha-1}},
\end{aligned}
$$

which is arbitrary small for large $|x|$. This proves the last assertion.

\section{Asymptotics and estimates of solutions}

First, we recall some results from [4] concerning $L^{p}$ estimates of the solutions to (1). We assume below that $\theta_{0} \in L^{\frac{2}{\alpha-1}}$. For $p \in\left[\frac{2}{\alpha-1}, \infty\right]$ we have (see [4, Prop. 3.2])

$$
t^{\frac{\alpha-1+|\mathbf{k}|}{\alpha}-\frac{2}{\alpha p}} \nabla^{\mathbf{k}} \theta \in B C\left((0, \infty), L^{p}\left(\mathbb{R}^{2}\right)\right),
$$

where $\mathbf{k} \in \mathbb{N} \times \mathbb{N}$. In particular, for $p \in\left[\frac{2}{\alpha-1}, \infty\right]$,

$$
\|\theta(t, \cdot)\|_{p} \lesssim t^{-\frac{\alpha-1}{\alpha}+\frac{2}{\alpha p}}, \quad t>0 .
$$

Combining this with (15), we get for $p \in\left[\frac{2}{\alpha-1}, \infty\right)$,

$$
\left\|R^{\perp} \theta(t, \cdot)\right\|_{p} \lesssim t^{-\frac{\alpha-1}{\alpha}+\frac{2}{\alpha p}}, \quad t>0 .
$$

We will need the following auxiliary lemma.

Lemma 3.1. Let $p \geq \frac{2}{\alpha-1}$ and $q=\frac{p}{p-1}$. Assume that $f(s, \cdot) \in L^{q}$ and $g(s, \cdot) \in L^{p}$ satisfy

$$
\|f(s, \cdot)\|_{q} \leq c_{1} s^{-\frac{3}{\alpha}+\frac{2}{\alpha q}}, \quad\|g(s, \cdot)\|_{p} \leq c_{2} s^{-\frac{\alpha-1}{\alpha}+\frac{2}{\alpha p}} .
$$

Then, there is a constant $C$ such that for $p \geq \frac{2}{\alpha-1}$,

$$
\int_{\mathbb{R}^{2}} \mid f\left(t-s, x-y|| g(s, y) \mid d y \leq C(t-s)^{-\left(\frac{1}{\alpha}+\frac{2}{p \alpha}\right)} s^{-\frac{\alpha-1}{\alpha}+\frac{2}{\alpha p}}, \quad 0<s<t, x \in \mathbb{R}^{2} .\right.
$$

Furthermore, for $t>0, x \in \mathbb{R}^{2}$ and $p>\frac{2}{\alpha-1}$, we have

$$
\int_{0}^{t} \int_{\mathbb{R}^{2}}|f(t-s, x-y)| g(s, y) \mid s^{-\frac{\alpha-1}{\alpha}} d y d s \leq C \mathcal{B}\left(\frac{p(\alpha-1)-2}{p \alpha}, \frac{p(2-\alpha)+2+p}{\alpha p}\right) t^{-\frac{\alpha-1}{\alpha}}
$$


Proof. By Hölder inequality,

$$
\int_{\mathbb{R}^{2}}\left|f(t-s, x-y)\|g(s, y) \mid d y \leq\| f(t-s, \cdot)\left\|_{\frac{p}{p-1}}\right\| g(s, \cdot) \|_{p} \leq c_{1} c_{2}(t-s)^{-\frac{1}{\alpha}-\frac{2}{\alpha p}} s^{-\frac{\alpha-1}{\alpha}+\frac{2}{\alpha p}}\right.
$$

which gives (23). Furthermore, this implies

$$
\begin{aligned}
\int_{0}^{t} \int_{\mathbb{R}^{2}}|f(t-s, x-y)| g(s, y) \mid s^{-\frac{\alpha-1}{\alpha}} d y d s & \leq c \int_{0}^{t}(t-s)^{-\frac{1}{\alpha}-\frac{2}{p \alpha}} s^{-\frac{2 \alpha-2}{\alpha}+\frac{2}{\alpha p}} d s \\
& =c t^{-\frac{\alpha-1}{\alpha}} \int_{0}^{1}(1-u)^{-\frac{1}{\alpha}-\frac{2}{p \alpha}} u^{-\frac{2 \alpha-2}{\alpha}+\frac{2}{\alpha p}} d u \\
& =c \mathcal{B}\left(\frac{p(\alpha-1)-2}{p \alpha}, \frac{(2-\alpha) p+2}{p \alpha}\right) t^{-\frac{\alpha-1}{\alpha}} .
\end{aligned}
$$

The next corollary is an immediate consequence of Lemma 3.1 .

Corollary 3.2. Let $\theta$ be a solution to (1) with $\theta_{0} \in L^{\frac{2}{\alpha-1}}$. For every $t>0$ and $p \in\left(\frac{2}{\alpha-1}, \infty\right)$, we have

$$
\begin{gathered}
\int_{0}^{t} \int_{\mathbb{R}^{2}}|\nabla p(t-s, \cdot)(x-y)||R \theta(s, y)| s^{-\frac{\alpha-1}{\alpha}} d y d s \leq C t^{-\frac{\alpha-1}{\alpha}}, \\
\int_{0}^{t} \int_{\mathbb{R}^{2}}|R \nabla p(t-s, \cdot)(x-y)||R \theta(s, y)| s^{-\frac{\alpha-1}{\alpha}} d y d s \leq C t^{-\frac{\alpha-1}{\alpha}} .
\end{gathered}
$$

Proof. Both of the bounds follow from (10), (15), (20) and (22) applied to (24).

The below-given bound extends (22) to $p \in(1, \infty]$.

Proposition 3.3. Assume $\theta_{0} \in L^{\frac{2}{\alpha-1}}$. There is a constant $C>0$ sucht that

$$
\left\|R^{\perp} \theta(t, \cdot)\right\|_{\infty} \leq C t^{-\frac{\alpha-1}{\alpha}} .
$$

Proof. By (2), we get

$$
R_{i} \theta(t, \cdot)(x)=R_{i} P_{t} \theta_{0}(x)+\int_{0}^{t} \int_{\mathbb{R}^{2}} R_{i} \nabla p(t-s, \cdot)(x-y) \cdot R^{\perp} \theta(s, y) \theta(s, y) d y d s .
$$

By Proposition 2.3, we have $\left\|R_{i} P_{t} \theta_{0}\right\|_{\infty} \leq c t^{-\frac{\alpha-1}{\alpha}}$ and the assertion follows from (21) and (26).

Now, we will pass to the proof of pointwise upper bounds for solutions to (11). Let $L^{p, \lambda}\left(\mathbb{R}^{2}\right)$ be the Morrey space, i.e.

$$
L^{p, \lambda}\left(\mathbb{R}^{2}\right)=\left\{f \in L^{p}\left(\mathbb{R}^{2}\right):\|f\|_{L^{p, \lambda}}:=\sup _{r>0} \sup _{x \in \mathbb{R}^{2}} r^{-\lambda} \int_{B(x, r) \cap \Omega}|f(z)|^{p} d z<\infty\right\} .
$$

The Morrey space is a Banach space with the norm $\|f\|_{L^{p, \lambda}}$. For any Banach space $X$ we denote by $L^{p, \lambda}((0, \infty) ; X)$ the space of functions $f:(0, \infty) \rightarrow X$ such that

$$
\|f\|_{L^{p, \lambda}((0, \infty) ; X)}:=\sup _{0<s<t<\infty}\left((t-s)^{-\lambda} \int_{s}^{t}\|f(r)\|_{X}^{p} d r\right)^{\frac{1}{p}}<\infty .
$$

It is also a Banach space with the norm $\|f\|_{L^{p, \lambda}((0, \infty) ; X)}$.

Lemma 3.4. Let $\theta_{0} \in L^{\frac{2}{\alpha-1}}\left(\mathbb{R}^{2}\right)$. There is a constant $C>0$ such that for all $t>0$ and $x \in \mathbb{R}^{2}$, we have

$$
\theta(t, x) \leq C P_{t}\left|\theta_{0}\right|(x)
$$


Proof. Let $v=R^{\perp} \theta$ and consider the linear equation

$$
\partial_{t} u=\Delta^{\alpha / 2} u+v \cdot \nabla u \text {. }
$$

By [16, Corollary 1.4], the fundamental solution $\tilde{p}(t, x, y)$ of (29) is bounded by $p(t, x-y)$, that is

$$
\tilde{p}(t, x, y) \leq c p(t, x-y), \quad t>0, x, y \in \mathbb{R}^{2} .
$$

Indeed, taking $\lambda=\frac{2(2-\alpha)}{\alpha}$ and $q=\infty$ in [16, Corollary 1.4], we only need to show that all required assumptions are satisfied, i.e. $\nabla v=0$ and

$$
\begin{aligned}
& v \in L^{2, \frac{2}{\alpha}-\frac{\lambda}{2}}\left((0, \infty) ; \mathcal{L}^{\frac{4}{\alpha}, \lambda}\left(\mathbb{R}^{2}\right)\right), \\
& v \in L_{l o c}^{\infty}\left((0, \infty) ; L_{l o c}^{1}\left(\mathbb{R}^{2}\right)\right), \\
& v \in L^{1, \frac{1}{\alpha}}\left((0, \infty) ; L_{u l o c}^{1}\left(\mathbb{R}^{2}\right)\right),
\end{aligned}
$$

where $\mathcal{L}^{p, \lambda}\left(\mathbb{R}^{2}\right)$ is a Campanato space. Since $\lambda=\frac{2(2-\alpha)}{\alpha}<2$ for $\alpha>1$, the Campanato space $\mathcal{L}^{\frac{4}{\alpha}, \lambda}\left(\mathbb{R}^{2}\right)$ reduces to the Morrey space $L^{\frac{4}{\alpha}, \lambda}\left(\mathbb{R}^{2}\right)$, see, e.g. [21]. Clearly, we have $\nabla v=0$. Furthermore, by (15), (21) and Hölder inequality,

$$
\begin{aligned}
& \left\|R^{\perp} \theta(u, \cdot)\right\|_{L^{\frac{4}{\alpha}}, \frac{2(2-\alpha)}{\alpha}} \leq \sup _{x \in \mathbb{R}^{2}, r>0}\left(r^{-\frac{2(2-\alpha)}{\alpha}} \int_{B(x, r)}|R \theta(t, z)|^{\frac{4}{\alpha}} d z\right)^{\frac{\alpha}{4}} \\
& \leq \sup _{x \in \mathbb{R}^{2}, r>0}\left(r^{-\frac{2(2-\alpha)}{\alpha}}\left(\int_{B(x, r)} d z\right)^{\frac{2-\alpha}{\alpha}}\left(\int_{\mathbb{R}^{2}}|R \theta(t, z)|^{\frac{2}{\alpha-1}} d z\right)^{\frac{2(\alpha-1)}{\alpha}}\right)^{\frac{\alpha}{4}} \\
& \leq c\left\|\theta_{0}\right\|_{\frac{2}{\alpha-1}} \text {. }
\end{aligned}
$$

Hence,

$$
\|v\|_{L^{2, \frac{2}{\alpha}-\frac{\lambda}{2}}\left((0, \infty) ; L^{\frac{4}{\alpha}}, \frac{2(2-\alpha)}{\alpha}\right)}=\sup _{t>0} \sup _{0<s<t}\left((t-s)^{-1} \int_{s}^{t}\|R \theta(u, \cdot)\|_{L^{\frac{4}{\alpha}}, \frac{2(2-\alpha)}{\alpha}}^{2} d u\right)^{\frac{1}{2}} \leq c\left\|\theta_{0}\right\|_{\frac{2}{\alpha-1}},
$$

which gives (31). Next, (32) is an immediate consequence of (27). Finally, by (27), we have

$$
\left\|R^{\perp} \theta(t, \cdot)\right\|_{L_{u l o c}^{1}\left(\mathbb{R}^{2}\right)}:=\sup _{x \in \mathbb{R}^{2}} \int_{B(x, 1)}\left|R^{\perp} \theta(t, y)\right| d y \lesssim\left\|R^{\perp} \theta\right\|_{\infty} \lesssim t^{-(\alpha-1) / \alpha} .
$$

Consequently,

$\sup _{t>0} \sup _{0<s<t}\left((t-s)^{-1 / \alpha} \int_{s}^{t}\|R \theta(u, \cdot)\|_{L_{u}^{1} \text { loc }}\left(\mathbb{R}^{2}\right) d u\right) \lesssim \sup _{t>0} \sup _{0<s<t}\left((t-s)^{-1 / \alpha} \int_{0}^{t-s} u^{-(\alpha-1) / \alpha} d u\right) \leq \alpha$,

which yields (33). Now consider (29) with initial condition $u_{0}=\theta_{0}$. Clearly,

$$
\theta(t, x)=\int_{\mathbb{R}^{2}} \tilde{p}(t, x, y) \theta_{0}(y) d y
$$

is a solution to this problem and (30) gives us

$$
|\theta(t, x)| \leq \int_{\mathbb{R}^{2}} \tilde{p}(t, x, y)\left|\theta_{0}(y)\right| d y \leq c \int_{\mathbb{R}^{2}} p(t, x, y)\left|\theta_{0}(y)\right| d y=c P_{t}\left|\theta_{0}\right|(x) .
$$

Proposition 3.5. Assume $\theta_{0} \in L^{\frac{2}{\alpha-1}}\left(\mathbb{R}^{2}\right)$. We have

$$
\lim _{t \rightarrow 0}\left\|t^{\frac{\alpha-1}{\alpha}} R \theta(t, \cdot)\right\|_{\infty}=\lim _{t \rightarrow \infty}\left\|t^{\frac{\alpha-1}{\alpha}} R \theta(t, \cdot)\right\|_{\infty}=\lim _{|x| \rightarrow \infty} \sup _{t>0}\left|t^{\frac{\alpha-1}{\alpha}} R \theta(t, x)\right|=0 .
$$


Proof. We will use the equality (2). The required results for the term $R_{i} P_{t} \theta_{0}(x)$ have been provided in Proposition 2.3. so what has left is to deal with the integral term. By (28) and (12), for every $\delta>0$ there are $t_{\delta}, T_{\delta}>0$ such that $\|\theta(s, \cdot)\|_{\infty}<\delta s^{-(\alpha-1) / \alpha}$ for $s<t_{\delta}$ or $s>T_{\delta}$. We fix some $p>\frac{2}{\alpha-1}$. Consequently, by (26),

$$
\left|\int_{0}^{t} \int_{\mathbb{R}^{2}} R_{i} \nabla p(t-s, x, y) \cdot R^{\perp} \theta_{s}(y) \theta(s, y) d y d s\right| \leq \delta c t^{-\frac{\alpha-1}{\alpha}}, \quad x \in \mathbb{R}^{d}, t \leq t_{\delta},
$$

which gives the first limit in (34). Now, let $t>2 T_{\delta}$. By (26), we get

$$
\left|\int_{T_{\delta}}^{t} \int_{\mathbb{R}^{2}} R_{i} \nabla p(t-s, x, y) \cdot R^{\perp} \theta_{s}(y) \theta(s, y) d y d s\right| \leq \delta c t^{-\frac{\alpha-1}{\alpha}}, \quad x \in \mathbb{R}^{d}, t>T_{\delta} .
$$

Next, by (23) (with $f=R_{i} \nabla p$ and $g=\theta R^{\perp} \theta$ ) and (21),

$$
\begin{aligned}
\left|\int_{0}^{T_{\delta}} \int_{\mathbb{R}^{2}} R_{i} \nabla p(t-s, x, y) \cdot R^{\perp} \theta(s, y) \theta(s, y) d y d s\right| & \lesssim \int_{0}^{T_{\delta}}(t-s)^{-\frac{1}{\alpha}-\frac{2}{\alpha p}} s^{-\frac{2(\alpha-1)}{\alpha}+\frac{2}{\alpha p}} d s \\
& \lesssim t^{-\frac{1}{\alpha}-\frac{2}{\alpha p}} \int_{0}^{T_{\delta}} s^{-1+\frac{p(2-\alpha)+2}{\alpha p}} d s \\
& =c t^{-\frac{\alpha-1}{\alpha}-\frac{p(2-\alpha)+2}{\alpha p}} .
\end{aligned}
$$

This proves the second limit in (34). Finally, we deal with $\lim _{|x| \rightarrow \infty} \sup _{t>0}\left|t^{\frac{\alpha-1}{\alpha}} R \theta(t, x)\right|=0$. By (28) and (14), for every $\varepsilon \in(0,1)$ there exists $r_{\varepsilon}$ such that $\sup _{s>0}\left|s^{\frac{\alpha-1}{\alpha}} \theta(s, y)\right|<\varepsilon$ for $|y|>r_{\varepsilon}$. Then, by (26),

$$
\left|\int_{0}^{t} \int_{B\left(0, r_{\varepsilon}\right)^{c}} R_{i} \nabla p(t-s, x, y) \cdot R^{\perp} \theta(s, y) \theta(s, y) d y d s\right| \leq \varepsilon c t^{-\frac{\alpha-1}{\alpha}} .
$$

Furthermore, by (16),

$$
\left|R_{i} \nabla p(t-s, x, y)\right| \leq c(t-s)^{-\frac{1}{\alpha}}|x-y|^{-2}<\varepsilon r_{\varepsilon}^{-2}(t-s)^{-\frac{1}{\alpha}}
$$

for $y \in B\left(0, r_{\varepsilon}\right)$ and $|x|$ sufficiently large. Hence, by (21) and (27), we get

$$
\begin{aligned}
& \left|\int_{0}^{t} \int_{B\left(0, r_{\varepsilon}\right)} R_{i} \nabla p(t-s, x, y) \cdot R^{\perp} \theta(s, y) \theta(s, y) d y d s\right| \leq \varepsilon r_{\varepsilon}^{-2} \int_{0}^{t} \int_{B\left(0, r_{\varepsilon}\right)}(t-s)^{-\frac{1}{\alpha}} s^{-\frac{2 \alpha-2}{\alpha}} d y d s \\
& \leq c \varepsilon t^{-\frac{\alpha-1}{\alpha}} \text {, }
\end{aligned}
$$

which ends the proof.

Proof of Theorem 1.2. First, observe that by (28) and semi-group property of $p(t, x)$,

$$
\begin{aligned}
& \left|\int_{0}^{t} \int_{\mathbb{R}^{2}} \nabla p(t-s, x-y) \cdot R \theta(s, y) \theta(s, y) d y d s\right| \\
& \lesssim \int_{0}^{t}(t-s)^{-\frac{1}{\alpha}}\|R \theta(s, \cdot)\|_{\infty} \int_{\mathbb{R}^{2}} p(t-s, x-y) P_{s} \theta_{0}(y) d y d s \\
& =P_{t} \theta_{0}(x) \int_{0}^{t}(t-s)^{-\frac{1}{\alpha}}\|R \theta(s, \cdot)\|_{\infty} d s .
\end{aligned}
$$

By Proposition 3.5. for every $\varepsilon>0$ there are $t_{\varepsilon}>0$ and $T_{\varepsilon}$ such that $\|R \theta(t, \cdot)\|_{\infty} \leq \varepsilon t^{-(\alpha-1) / \alpha}$ for $t<t_{\varepsilon}$ or $t>T_{\varepsilon}$. Hence, by (36), for $t<t_{\varepsilon}$, we have

$$
\begin{aligned}
\left|\int_{0}^{t} \int_{\mathbb{R}^{2}} \nabla p(t-s, x-y) \cdot R \theta(s, y) \theta(s, y) d y d s\right| & \leq \varepsilon P_{t} \theta_{0}(x) \int_{0}^{t}(t-s)^{-\frac{1}{\alpha}} s^{-\frac{\alpha-1}{\alpha}} d s \\
& =\varepsilon P_{t} \theta_{0}(x) \mathcal{B}\left(\frac{\alpha-1}{\alpha}, \frac{1}{\alpha}\right)
\end{aligned}
$$


Thus, (2) gives us

$$
\left|\frac{\theta(t, x)}{P_{t} \theta_{0}(x)}-1\right| \lesssim \varepsilon
$$

which proves the first limit in (5). Similarly, we get for $t>2 T_{\varepsilon}$,

$$
\begin{aligned}
& \left|\int_{0}^{t} \int_{\mathbb{R}^{2}} \nabla p(t-s, x-y) \cdot R \theta_{s}(y) \theta(s, y) d y d s\right| \\
& \leq P_{t} \theta_{0}(x)\left(c \int_{0}^{T_{\varepsilon}}(t-s)^{-\frac{1}{\alpha}} s^{-\frac{\alpha-1}{\alpha}} d s+\varepsilon \int_{T_{\varepsilon}}^{t}(t-s)^{-\frac{1}{\alpha}} s^{-\frac{\alpha-1}{\alpha}} d s\right) \\
& \lesssim P_{t} \theta_{0}(x)\left(c t^{\left.-\frac{1}{\alpha} T_{\varepsilon}^{\frac{1}{\alpha}}+\varepsilon \mathcal{B}\left(\frac{\alpha-1}{\alpha}, \frac{1}{\alpha}\right)\right),}\right.
\end{aligned}
$$

which is less than $2 \varepsilon \mathcal{B}\left(\frac{\alpha-1}{\alpha}, \frac{1}{\alpha}\right) P_{t} \theta_{0}(x)$ for $t$ large enough. Hence, we obtain the second limit in (5).

Finally, the previous limit lets us prove $\lim _{|x| \rightarrow \infty} \sup _{t>0}\left|\frac{\theta(t, x)}{P_{t} \theta_{0}(x)}-1\right|=0$ by showing that

$$
\lim _{|x| \rightarrow \infty} \sup _{0<t<T}\left|\frac{\theta(t, x)}{P_{t} \theta_{0}(x)}-1\right|=0
$$

holds for any $T>0$. By (34), for every $\varepsilon>0$ there is $M>0$ such that $\left|t^{\frac{\alpha-1}{\alpha}} R^{\perp} \theta(t, x)\right|<\varepsilon$ for $|x|>M$. Hence, by (91) and (28), we get

$$
\begin{aligned}
& \left|\int_{0}^{t} \int_{|y|>M} \nabla p(t-s, x-y) \cdot R \theta(s, y) \theta(s, y) d y d s\right| \\
& \lesssim \varepsilon \int_{0}^{t}(t-s)^{-\frac{1}{\alpha}} s^{-\frac{\alpha-1}{\alpha}} \int_{\mathbb{R}^{2}} p(t-s, x-y) P_{s} \theta_{0}(y) d y d s=\varepsilon \mathcal{B}\left(\frac{\alpha-1}{\alpha}, \frac{1}{\alpha}\right) P_{t} \theta_{0}(x) .
\end{aligned}
$$

Next, for $|x|>2 M$ and $t<T$, by (9),

$$
\begin{aligned}
& \left|\int_{0}^{t} \int_{|y| \leq M} \nabla p(t-s, x-y) \cdot R \theta(s, y) \theta(s, y) d y d s\right| \\
& \lesssim\left|\int_{0}^{t} s^{-(\alpha-1) / \alpha} \int_{|y| \leq M} \frac{1}{|x|^{\frac{1}{\alpha}}} p(t-s, x-y) P_{s} \theta_{0}(y) d y d s\right| \leq \frac{\alpha T^{\frac{1}{\alpha}}}{|x|^{\frac{1}{\alpha}}} P_{t} \theta_{0}(x) .
\end{aligned}
$$

This ends the proof.

Proof of Theorem 1.1. The upper bound follows from Lemma 3.4. To prove the lower one, note that Lemma 1.2 implies $\theta(t, x) \gtrsim P_{t} \theta_{0}(x)$ whenever $t \in\left(0, t_{0}\right) \cup(T, \infty)$ or $|x|>R$ for some $t_{0}, T, R>0$. Since both, $\theta(t, x)$ and $P_{t} \theta_{0}(x)$ are continuous, they are comparable on $\overline{\left[t_{0}, T\right] \times B(0, R)}$ as well.

In the last part of this section, we consider the case $\theta_{0} \in L^{p}$ with $p>\frac{2}{\alpha-1}$. As a result, we obtain the local in time analog of Theorem 1.1. By Remark 3.3 in [4], for $p>\frac{2}{\alpha-1}$, we have

$$
\|\theta(t, \cdot)\|_{q} \lesssim t^{-\frac{2}{\alpha}\left(\frac{1}{p}-\frac{1}{q}\right)}, \quad p \leq q \leq \infty .
$$

Proposition 3.6. For nonnegative $\theta_{0} \in L^{p}\left(\mathbb{R}^{2}\right), p>\frac{2}{1-\alpha}$ and $T>0$ there are constants $C_{1}$ and $C_{2}$ (depending on $T$ ) such that

$$
C_{1} P_{t} \theta_{0}(x) \leq \theta(t, x) \leq C_{2} P_{t} \theta_{0}(x), \quad x \in \mathbb{R}^{2}, 0<t \leq T .
$$

Proof. Let $T>0$. Let us consider the equation

$$
\begin{cases}\partial_{t} u & =\Delta^{\alpha / 2} u+b(t, x) \cdot \nabla u \\ u(0, x) & =\theta_{0}(x)\end{cases}
$$


where $b(t, x)=\left(R^{\perp} \theta\right)(t, x)$. Of course $u(t, x)=\theta(t, x)$ is a solution to the above equation. By (15), we have

$$
\|b(t, \cdot)\|_{p} \leq c\|\theta(t, \cdot)\|_{p} \leq c\left\|\theta_{0}\right\|_{p}
$$

By Hölder inequality,

$$
\begin{aligned}
& \int_{s}^{t} \int_{\mathbb{R}^{2}} \frac{p(u-s, z-x)}{(u-s)^{1 / \alpha}}|b(u, z)| d z d u \leq \int_{s}^{t} \frac{1}{(u-s)^{1 / \alpha}}\|p(u-s, \cdot)\|_{\frac{p}{p-1}}\|b(u, \cdot)\|_{p} d u \\
& \leq c \int_{s}^{t} \frac{1}{(u-s)^{1 / \alpha}}(u-s)^{\frac{2}{\alpha}\left(\frac{p-1}{p}-1\right)} d u=c \int_{s}^{t}(u-s)^{-\frac{2}{\alpha p}-\frac{1}{\alpha}} d u=c_{1}(t-s)^{1-\frac{2+p}{\alpha p}} .
\end{aligned}
$$

In the same way, we get

$$
\int_{s}^{t} \int_{\mathbb{R}^{2}} \frac{p(t-u, z-x)}{(t-u)^{1 / \alpha}}|b(u, z)| d z d u \leq c_{1}(t-s)^{1-\frac{2+p}{\alpha p}} .
$$

Note that $\frac{2+p}{\alpha p}<1$, and consequently $c(t-s)^{1-\frac{2+q}{\alpha q}} \leq \eta+\beta(t-s)$ for arbitrary small $\eta$ and some $\beta>0$. Hence, $b$ belongs to the class $\mathcal{K}(\eta, Q)$ (see [13, Definition 1]. Then, by [13, Theorems 2 and 3], the fundamental solution of the equation $\partial_{t} u=\Delta^{\alpha / 2} u+b(t, x) \cdot \nabla u$ is locally in time comparable with $p$ and we get the assertion of the proposition.

\section{Gradient estimates}

In this section we derive the pointwise estimates for $\nabla^{\mathbf{k}} \theta$. Recall that for a multi-index $\mathbf{k}=\left(k_{1}, k_{2}\right) \in \mathbb{N}^{2}$, we put $|\mathbf{k}|=k_{1}+k_{2}$. Note that

$$
\nabla^{\mathbf{k}}(f g)=\sum_{\mathbf{m}+\mathbf{n}=\mathbf{k}} c_{\mathbf{m}, \mathbf{n}} \nabla^{\mathbf{m}} f \nabla^{\mathbf{n}} g
$$

where the sum is taken over all multi-indices $\mathbf{m}$ and $\mathbf{n}$ such that $\mathbf{m}+\mathbf{n}=\mathbf{k}$.

Lemma 4.1. For $\theta_{0} \in L^{\frac{2}{\alpha-1}}$, we have

$$
\left\|\nabla^{\mathbf{k}} R_{i} \theta(t, \cdot)\right\|_{\infty} \lesssim t^{-\frac{|\mathbf{k}|+\alpha-1}{\alpha}}, \quad i=1,2 .
$$

Proof. Let us rewrite (2) as follows,

$$
\begin{aligned}
\theta(t, \cdot)(x) & =\int_{\mathbb{R}^{2}} p(t, x-y) \theta_{0}(y) d y+\int_{0}^{t / 2} \int_{\mathbb{R}^{2}} \nabla p(t-s, x-y) \cdot R^{\perp} \theta(s, y) \theta(s, y) d y d s \\
& +\int_{t / 2}^{t} \int_{\mathbb{R}^{2}} \nabla p(t-s, y) \cdot R^{\perp} \theta(s, x-y) \theta(s, x-y) d y d s .
\end{aligned}
$$

Since the Riesz transform commutes with derivatives, by (39) and (37), we get

$$
\begin{aligned}
& \nabla^{\mathbf{k}} R_{i} \theta(t, x) \\
&(40)= \int_{\mathbb{R}^{2}} R_{i} \nabla^{\mathbf{k}} p(t, x-y) \theta_{0}(y) d y+\int_{0}^{t / 2} \int_{\mathbb{R}^{2}} R_{i}\left(\nabla^{\mathbf{k}} \nabla p(t-s, x-y)\right) \cdot R^{\perp} \theta(s, y) \theta(s, y) d y d s \\
&+\sum_{\mathbf{k}_{\mathbf{1}}+\mathbf{k}_{\mathbf{2}}=\mathbf{k}} c_{\mathbf{k}_{\mathbf{1}}, \mathbf{k}_{\mathbf{2}}} \int_{t / 2}^{t} \int_{\mathbb{R}^{2}} R_{i}(\nabla p(t-s, y)) \cdot R^{\perp}\left(\nabla^{\mathbf{k}_{1}} \theta(s, x-y)\right) \nabla^{\mathbf{k}_{2}} \theta(s, x-y) d y d s,
\end{aligned}
$$


where $\mathbf{k}_{\mathbf{1}}, \mathbf{k}_{\mathbf{2}} \in \mathbb{N}^{2}$. Hence, by Hölder inequality, (21), (10), (15) and (20), for $p>\frac{2}{\alpha-1}$, we get

$$
\begin{aligned}
\left\|\nabla^{\mathbf{k}} R_{i} \theta(t, \cdot)\right\|_{\infty} \lesssim & \left\|\nabla^{\mathbf{k}} p(t, \cdot)\right\|_{\frac{2}{3-\alpha}}\left\|\theta_{0}\right\|_{\frac{2}{\alpha-1}} \\
& +\int_{0}^{t / 2} s^{-\frac{\alpha-1}{\alpha}}\left\|\nabla^{\mathbf{k}} \nabla p(t-s, \cdot)\right\|_{\frac{2}{3-\alpha}}\|\theta(s, \cdot)\|_{\frac{2}{\alpha-1}} d s \\
& +\sum_{\mathbf{k}_{\mathbf{1}}+\mathbf{k}_{\mathbf{2}}=\mathbf{k}} c_{\mathbf{k}_{\mathbf{1}}, \mathbf{k}_{\mathbf{2}}} \int_{t / 2}^{t}\|\nabla p(t-s, \cdot)\|_{\frac{p}{p-1}} \|\left(R^{\perp} \nabla^{\mathbf{k}_{\mathbf{1}}} \theta(s, \cdot)\left\|_{p}\right\| \nabla^{\mathbf{k}_{\mathbf{2}}} \theta(s, \cdot) \|_{\infty} d s\right. \\
\lesssim & t^{-\frac{|\mathbf{k}|+\alpha-1}{\alpha}}+\int_{0}^{t / 2} s^{-\frac{\alpha-1}{\alpha}} t^{-\frac{|\mathbf{k}|+1+\alpha}{\alpha}} d s \\
& +\sum_{\mathbf{k}_{\mathbf{1}}+\mathbf{k}_{\mathbf{2}}=\mathbf{k}} c_{\mathbf{k}_{\mathbf{1}}, \mathbf{k}_{\mathbf{2}}} \int_{t / 2}^{t}(t-s)^{-\frac{1}{\alpha}-\frac{2}{\alpha p}} t^{-\frac{\left|\mathbf{k}_{\mathbf{1}}\right|}{\alpha}-\frac{\alpha-1}{\alpha}+\frac{2}{\alpha p}} t^{-\frac{\left|\mathbf{k}_{\mathbf{2}}\right|+\alpha-1}{\alpha}} d s \\
\lesssim & t^{-\frac{|\mathbf{k}|+\alpha-1}{\alpha}}
\end{aligned}
$$

as required.

Next, we present a series of auxiliary lemmas that are used in the proof of Theorem 1.3.

Lemma 4.2. Let $0<t_{1}<t_{2}<\infty$ and $\theta_{0} \in L^{\frac{2}{\alpha-1}}$. If $\left\|\theta_{0}\right\|_{\frac{2}{\alpha-1}}>0$, then, there exists a constant $C=C\left(t_{1}, t_{2}, \theta_{0}\right)$ such that

$$
P_{t}\left|\theta_{0}(x)\right| \geq \frac{C}{(1+|x|)^{2+\alpha}}, \quad t_{2}>t>t_{1}, x \in \mathbb{R}^{2} .
$$

Proof. Since $\theta_{0} \in L^{\frac{2}{\alpha-1}}$, then $\theta_{0} \in L_{l o c}^{1}$. Hence, there is $R>0$ such that $C<\int_{B(0, R)}\left|\theta_{0}(y)\right| d y<$ $\infty$ for some $c>0$. By (8), we get

$$
\begin{aligned}
P_{t}\left|\theta_{0}\right|(x) \geq \int_{B(0, R)} p(t, x-y)\left|\theta_{0}(y)\right| d y & \geq c_{1} \frac{t}{\left(t^{1 / \alpha}+2 R+|x|\right)^{2+\alpha}} \int_{B(0, R)}\left|\theta_{0}(y)\right| d y \\
& \geq c c_{1} \frac{t_{1}}{\left(2 t_{2}^{1 / \alpha}+2 R+|x|\right)^{2+\alpha}} \geq \frac{C}{(1+|x|)^{2+\alpha}} .
\end{aligned}
$$

Lemma 4.3. Let $\theta_{0} \in L^{\frac{2}{\alpha-1}}$. Let $0<t_{1}<t_{2}<\infty$. There exists a constant $C$ depending on $t_{1}, t_{2}, R$ and $\theta_{0}$ such that for $x \in \mathbb{R}^{2}$, we have

$$
\int_{D_{t}} \int_{B(0, R)}(t-s)^{-1 / \alpha} p(t-s, x-y) s^{-(\alpha-1) / \alpha}\left|\nabla^{\mathbf{k}} \theta(s, y)\right| d y d s \leq C t^{-|\mathbf{k}| / \alpha} P_{t}\left|\theta_{0}\right|(x),
$$

where $D_{t}=\left(t_{1}, t_{2}\right) \cap(t / 2, t)$.

Proof. Note that $D_{t}=\emptyset$ for $t \notin\left(t_{1}, 2 t_{2}\right)$, hence, it suffices to consider only $t_{1}<t<2 t_{2}$. By (20),

$$
\begin{aligned}
& \int_{D_{t}} \int_{B(0, R)}(t-s)^{-\frac{1}{\alpha}} p(t-s, x-y) s^{-(\alpha-1) / \alpha}\left|\nabla^{\mathbf{k}} \theta(s, y)\right| d y d s \\
& \leq c \int_{D_{t}} \int_{B(0, R)}(t-s)^{-\frac{1}{\alpha}} p(t-s, x-y) s^{-\frac{\alpha-1}{\alpha}-\frac{\alpha-1}{\alpha}-\frac{|\mathbf{k}|}{\alpha}} d y d s \\
& \leq c t_{1}^{-\frac{2(\alpha-1)}{\alpha}}\left(\frac{t}{2}\right)^{-\frac{|\mathbf{k}|}{\alpha}} \int_{D_{t}}(t-s)^{-\frac{1}{\alpha}} P_{t-s} \mathbb{1}_{B(0, R)}(x) d s=: f(t, x) .
\end{aligned}
$$

Note that $p(s, y) \geq \frac{1}{c_{1}}>0$ for $(s, y) \in\left(t_{1}, t_{2}\right) \times B(0, R)$. Hence,

$$
P_{t-s} \mathbb{1}_{B(0, R)}(x) \leq c_{1} \int_{\mathbb{R}^{2}} p(t-s, x-y) p(s, y) d y=c_{1} p(t, x) \leq \frac{c_{2}}{(1+|x|)^{2+\alpha}}
$$


Consequently, by Lemma 4.2 .

$$
f(t, x) \leq c_{3} t^{-\frac{|\mathbf{k}|}{\alpha}} \int_{t_{1}}^{t}(t-s)^{-\frac{1}{\alpha}} \frac{1}{(1+|x|)^{2+\alpha}} d s \leq c_{4} t^{-\frac{|\mathbf{k}|}{\alpha}} P_{t}\left|\theta_{0}\right|(x)
$$

Lemma 4.4. Let $\beta>0$ be fixed. For any $v \in(0,1)$, we have

$$
\int_{v}^{1} r^{-\beta}\left(1-r^{\alpha}\right)^{-1 / \alpha}\left(r^{\alpha}-v^{\alpha}\right)^{-1 / \alpha} d r \approx v^{-\beta}(1-v)^{1-2 / \alpha}
$$

with comparability constants depending only on $\alpha$ and $\beta$.

Proof. Denote the above integral by $I(v)$. Since $a^{\gamma}-b^{\gamma} \approx(a-b) a^{\gamma-1}$ for $a>b>0$ and $\gamma>0$ (see e.g Lemma 4 in [17]), we have $1-r^{\alpha} \approx 1-r$ and $r^{\alpha}-v^{\alpha} \approx(r-v) r^{\alpha-1}$. Hence,

$$
I(v) \approx \int_{v}^{1} r^{1 / \alpha-1-\beta}(1-r)^{-1 / \alpha}(r-v)^{-1 / \alpha} d r .
$$

For $v \geq 1 / 4$, we estimate $r^{1 / \alpha-1-\beta} \approx 1$ and substitute $r=1-u(1-v)$, which gives us

$$
I(v) \approx(1-v)^{1-2 / \alpha} \int_{0}^{1} u^{-1 / \alpha}(1-u)^{-1 / \alpha} d u=c(1-v)^{1-2 / \alpha}
$$

In the case $v<1 / 4$, we split the integral into $\int_{v}^{1 / 2}+\int_{1 / 2}^{1}$ and obtain

$$
\begin{aligned}
I(v) & \approx \int_{v}^{1 / 2} r^{1 / \alpha-1-\beta}(r-v)^{-1 / \alpha} d r+\int_{1 / 2}^{1}(1-r)^{-1 / \alpha} d r \\
& =v^{-\beta} \int_{1}^{1 /(2 v)} u^{1 / \alpha-1-\beta}(u-1)^{-1 / \alpha} d u+\frac{\alpha 2^{(\alpha-1) / \alpha}}{\alpha-1} \\
& \approx v^{-\beta}+1 \approx v^{-\beta}
\end{aligned}
$$

which is equivalent to the required formula under current assumptions.

Since $\alpha>1$, we immediately obtain the following

Corollary 4.5. Let $\beta>0$ be fixed. There is a constant $C_{\beta}$ such that for $v \in(0,1)$, we have

$$
\int_{v}^{1} r^{-\beta}\left(1-r^{\alpha}\right)^{-1 / \alpha}\left(r^{\alpha}-v^{\alpha}\right)^{-1 / \alpha} d r \leq C_{\beta} v^{-\beta}(1-v)^{-1 / \alpha}
$$

Lemma 4.6. Fix $\gamma \in\left(0, \frac{1}{\alpha}\right)$. For any measurable function $f: \mathbb{R} \times \mathbb{R}^{2} \rightarrow \mathbb{R}$, define the operator

$$
T_{\gamma} f(t, x)=t^{\gamma} \int_{0}^{t} s^{-\gamma-\frac{\alpha-1}{\alpha}}(t-s)^{-\frac{1}{\alpha}} P_{t-s}|f|(s, x) d s .
$$

Suppose $T_{\gamma} f(t, x)<\infty$ and $f$ satisfies the inequality

$$
f(t, x) \leq C P_{t} \theta_{0}(x)+\eta T_{\gamma} f(t, x), \quad t>0, x \in \mathbb{R}^{2},
$$

for some constants $C, \eta>0$. If $\eta$ is sufficiently small, then there exists a constant $M>0$ such that

$$
f(t, x) \leq M P_{t}\left|\theta_{0}\right|(x), \quad t>0, x \in \mathbb{R}^{2}
$$


Proof. Applying estimate (42) of $f$ to (41), we get

$$
\begin{aligned}
T_{\gamma} f(t, x) \leq & t^{\gamma} \int_{0}^{t} s^{-\gamma-(\alpha-1) / \alpha}(t-s)^{-1 / \alpha} \int_{\mathbb{R}^{2}} p(t-s, x-y) \\
& \times\left(C P_{s}\left|\theta_{0}\right|(y)+\eta \int_{0}^{s} u^{-(\alpha-1) / \alpha}(s-u)^{-1 / \alpha} P_{s-u}|f|(u, y) d u\right) d y d s \\
= & C \mathcal{B}\left(1-\gamma-\frac{\alpha-1}{\alpha}, 1-\frac{1}{\alpha}\right) P_{t}\left|\theta_{0}\right|(x) \\
& +t^{\gamma} \eta \int_{0}^{t} \int_{0}^{s} s^{-\gamma}(s u)^{-(\alpha-1) / \alpha}[(t-s)(s-u)]^{-1 / \alpha} P_{t-u}|f|(u, x) d u d s \\
= & C \mathcal{B}\left(1-\gamma-\frac{\alpha-1}{\alpha}, 1-\frac{1}{\alpha}\right) P_{t}\left|\theta_{0}(x)\right| \\
& +\eta t^{\gamma} \int_{0}^{t} u^{-(\alpha-1) / \alpha} P_{t-u}|f|(u, x) \int_{u}^{t} s^{-\gamma-(\alpha-1) / \alpha}[(t-s)(s-u)]^{-1 / \alpha} d s d u
\end{aligned}
$$

where $\mathcal{B}$ is the beta function. Using Corollary 4.5 with $\beta=\gamma \alpha$ and $v=(u / t)^{1 / \alpha}$, we estimate the last inner integral in (43) as follows

$$
\begin{aligned}
& \int_{u}^{t} s^{-\gamma-(\alpha-1) / \alpha}[(t-s)(s-u)]^{-1 / \alpha} d s \\
& =t^{-\gamma-1 / \alpha} \int_{u / t}^{1} s^{-\gamma-(\alpha-1) / \alpha}\left[(1-s)\left(s-\frac{u}{t}\right)\right]^{-1 / \alpha} d s \\
& =t^{-\gamma-1 / \alpha} \int_{(u / t)^{1 / \alpha}}^{1} r^{-\gamma \alpha}\left[\left(1-r^{\alpha}\right)\left(r^{\alpha}-\frac{u}{t}\right)\right]^{-1 / \alpha} d s \\
& \leq c_{\gamma} u^{-\gamma}(t-u)^{-1 / \alpha} .
\end{aligned}
$$

This yields $T_{\gamma} f(t, x) \leq C \mathcal{B}\left(1-\gamma-\frac{\alpha-1}{\alpha}, 1-\frac{1}{\alpha}\right) P_{t}\left|\theta_{0}(x)\right|+\eta c_{\gamma} T_{\gamma} f(t, x)$. Now, for $\eta<\frac{1}{c_{\gamma}}$, we get

$$
T_{\gamma} f(t, x) \leq \frac{C \mathcal{B}\left(1-\gamma-\frac{\alpha-1}{\alpha}, 1-\frac{1}{\alpha}\right)}{1-\eta c_{\gamma}} P_{t}\left|\theta_{0}\right|(x),
$$

which ends the proof.

Proof of Theorem 1.3. We will use induction with respect to $|\mathbf{k}|$. For $|\mathbf{k}|=0$ the assertion is true by Lemma 3.4. Assume now that (16) holds for all multi-indices $\mathbf{k}^{\prime}$ such that $\left|\mathbf{k}^{\prime}\right| \leq|\mathbf{k}|-1$ for some multi-index $\mathbf{k},|\mathbf{k}| \geqslant 1$. We use (39) and, analogously as in (40), we obtain

$$
\begin{aligned}
\nabla^{\mathbf{k}} \theta(t, x)= & \nabla^{\mathbf{k}} P_{t} \theta_{0}(x)+\int_{0}^{t / 2} \int_{\mathbb{R}^{2}}\left(\nabla^{\mathbf{k}} \nabla p(t-s, x-y)\right) \cdot R^{\perp} \theta(s, y) \theta(s, y) d y d s \\
& +\sum_{\mathbf{k}_{\mathbf{1}}+\mathbf{k}_{\mathbf{2}}=\mathbf{k}} c_{\mathbf{k}_{1}, \mathbf{k}_{\mathbf{2}}} \int_{t / 2}^{t} \int_{\mathbb{R}^{2}}(\nabla p(t-s, x-y)) \cdot R^{\perp}\left(\nabla^{\mathbf{k}_{1}} \theta(s, y)\right) \nabla^{\mathbf{k}_{\mathbf{2}}} \theta(s, y) d y d s
\end{aligned}
$$

As mentioned in Introduction, (9) implies

$$
\left|\nabla^{\mathbf{k}} P_{t} \theta_{0}(x)\right| \leqslant \int_{\mathbb{R}^{2}}\left|\nabla^{\mathbf{k}} p(t, x-y) \theta_{0}(y)\right| d y \lesssim t^{-\frac{|\mathbf{k}|}{\alpha}} P_{t}\left|\theta_{0}\right|(x)
$$


Next, by (9), Proposition 3.3. Lemma 3.4 and semigroup property, we get

$$
\begin{aligned}
& \left|\int_{0}^{t / 2} \int_{\mathbb{R}^{2}}\left(\nabla^{\mathbf{k}} \nabla p(t-s, x-y)\right) \cdot R^{\perp} \theta(s, y) \theta(s, y) d y d s\right| \\
& \lesssim t^{-(|\mathbf{k}|+1) / \alpha} \int_{0}^{t / 2} s^{-(\alpha-1) / \alpha} \int_{\mathbb{R}^{2}} p(t-s, x-y) P_{s}\left|\theta_{0}\right|(y) d y d s \\
& =c t^{-\frac{|\mathbf{k}|}{\alpha}} P_{t}\left|\theta_{0}\right|(x) .
\end{aligned}
$$

Hence, using the induction assumption for $\left|\mathbf{k}_{\mathbf{2}}\right| \leq|\mathbf{k}|-1$ together with (9), (28), (38) and semi-group property of $p(t, x)$, we conclude

$$
\begin{aligned}
& \left|\nabla^{\mathbf{k}} \theta(t, x)\right| \lesssim t^{-\frac{|\mathbf{k}|}{\alpha}} P_{t}\left|\theta_{0}\right|(x)+\sum_{\substack{\mathbf{k}_{1}+\mathbf{k}_{2}=\mathbf{k} \\
\left|\mathbf{k}_{\mathbf{2}}\right| \leq|\mathbf{k}|-1}} c_{\mathbf{k}_{\mathbf{1}}, \mathbf{k}_{\mathbf{2}}} t^{-(|\mathbf{k}|+\alpha-1) / \alpha} \int_{t / 2}^{t}(t-s)^{-1 / \alpha} \int_{\mathbb{R}^{2}} p(t-s, x-y) P_{s}\left|\theta_{0}\right|(y) d y d s \\
& +\int_{t / 2}^{t} \int_{\mathbb{R}^{2}}\left|\nabla p(t-s, x-y) \cdot R^{\perp} \theta(s, y) \nabla^{\mathbf{k}} \theta(s, y)\right| d y d s \\
& \lesssim t^{-\frac{|\mathbf{k}|}{\alpha}} P_{t}\left|\theta_{0}\right|(x)+\int_{t / 2}^{t}(t-s)^{-\frac{1}{\alpha}} \int_{\mathbb{R}^{2}} p(t-s, x-y)\left|R^{\perp} \theta(s, y)\right|\left|\nabla^{\mathbf{k}} \theta(s, y)\right| d y d s .
\end{aligned}
$$

Let $\varepsilon>0$, to be fixed later. By (34), there are $t_{1}, t_{2}, R>0$ such that $\left|s^{(\alpha-1) / \alpha} R^{\perp} \theta(s, y)\right|<\varepsilon$ for $(s, y) \notin D=\left(t_{1}, t_{2}\right) \times B(0, R)$. Thus

$$
\begin{aligned}
\left|\nabla^{\mathbf{k}} \theta(t, x)\right| \leq & c t^{-|k| / \alpha} P_{t}\left|\theta_{0}\right|(x)+\varepsilon \int_{t / 2}^{t}(t-s)^{-1 / \alpha} \int_{\mathbb{R}^{2}} p(t-s, x-y) s^{-(\alpha-1) / \alpha}\left|\nabla^{\mathbf{k}} \theta(s, y)\right| d y d s \\
& +\int_{t_{1} \vee t / 2}^{t_{2} \wedge t} \int_{B(0, R)}(t-s)^{-1 / \alpha} p(t-s, x-y) s^{-(\alpha-1) / \alpha}\left|\nabla^{\mathbf{k}} \theta(s, y)\right| d y d s .
\end{aligned}
$$

By Lemma 4.3, the last integral is bounded by $t^{-|\mathbf{k}| / \alpha} P_{t} \theta_{0}(x)$. This gives us

$$
\left|\nabla^{\mathbf{k}} \theta(t, x)\right| \leq c t^{-|\mathbf{k}| / \alpha} P_{t}\left|\theta_{0}\right|(x)+\varepsilon \int_{t / 2}^{t}(t-s)^{-1 / \alpha} \int_{\mathbb{R}^{2}} p(t-s, x-y) s^{-(\alpha-1) / \alpha}\left|\nabla^{\mathbf{k}} \theta(s, y)\right| d y d s .
$$

Now, denote $f_{\mathbf{k}}(t, x)=t^{|\mathbf{k}| / \alpha}\left|\nabla^{\mathbf{k}} \theta(t, x)\right|$. Then, for any $\gamma \in(0,1 / \alpha)$,

$$
\begin{aligned}
f_{\mathbf{k}}(t, x) & \leq c P_{t}\left|\theta_{0}\right|(x)+\varepsilon \int_{t / 2}^{t}(t-s)^{-1 / \alpha} \int_{\mathbb{R}^{2}} p(t-s, x-y) s^{-(\alpha-1) / \alpha} t^{|\mathbf{k}| / \alpha}\left|\nabla^{\mathbf{k}} \theta(s, y)\right| d y d s \\
& \leq c P_{t}\left|\theta_{0}\right|(x)+\varepsilon 2^{|\mathbf{k}| / \alpha} \int_{t / 2}^{t}(t-s)^{-1 / \alpha} s^{-(\alpha-1) / \alpha} P_{t-s} f_{\mathbf{k}}(s, x) d s \\
& \leq c P_{t}\left|\theta_{0}\right|(x)+\varepsilon 2^{|\mathbf{k}| / \alpha} T_{\gamma} f_{\mathbf{k}}(t, x),
\end{aligned}
$$

where $T_{\gamma}$ is defined in Lemma 4.6. Since $\varepsilon$ may be choosen arbitrary small, by Lemma 4.6,

$$
\left|\nabla^{\mathbf{k}} \theta(t, x)\right| \leq M t^{-|\mathbf{k}| / \alpha} P_{t}\left|\theta_{0}\right|(x) .
$$

The proof is complete.

\section{REFERENCES}

[1] K. Bogdan, T. Byczkowski, T. Kulczycki, M. Ryznar, R. Song, and Z. Vondraček. Potential analysis of stable processes and its extensions, volume 1980 of Lecture Notes in Mathematics. Springer-Verlag, Berlin, 2009. Edited by Piotr Graczyk and Andrzej Stos.

[2] K. Bogdan, T. Grzywny, and M. Ryznar. Heat kernel estimates for the fractional Laplacian with Dirichlet conditions. Ann. Probab., 38(5):1901-1923, 2010.

[3] K. Bogdan and T. Jakubowski. Estimates of heat kernel of fractional Laplacian perturbed by gradient operators. Comm. Math. Phys., 271(1):179-198, 2007. 
[4] J. A. Carrillo and L. C. F. Ferreira. The asymptotic behaviour of subcritical dissipative quasi-geostrophic equations. Nonlinearity, 21(5):1001-1018, 2008.

[5] P. Constantin, A. J. Majda, and E. Tabak. Formation of strong fronts in the 2-D quasigeostrophic thermal active scalar. Nonlinearity, 7(6):1495-1533, 1994.

[6] P. Constantin and J. Wu. Behavior of solutions of 2D quasi-geostrophic equations. SIAM J. Math. Anal., 30(5):937-948, 1999.

[7] P. Constantin and J. Wu. Hölder continuity of solutions of supercritical dissipative hydrodynamic transport equations. Ann. Inst. H. Poincaré Anal. Non Linéaire, 26(1):159-180, 2009.

[8] A. Córdoba and D. Córdoba. A maximum principle applied to quasi-geostrophic equations. Comm. Math. Phys., 249(3):511-528, 2004.

[9] J. Duoandikoetxea. Fourier analysis, volume 29 of Graduate Studies in Mathematics. American Mathematical Society, Providence, RI, 2001. Translated and revised from the 1995 Spanish original by David Cruz-Uribe.

[10] T. Grzywny and K. Szczypkowski. Estimates of heat kernels of non-symmetric lévy processes. 2017.

[11] T. Jakubowski and G. Serafin. Pointwise estimates for solutions of fractal Burgers equation. J. Differential Equations, 261(11):6283-6301, 2016.

[12] T. Jakubowski and G. Serafin. Stable estimates for source solution of critical fractal Burgers equation. Nonlinear Anal., 130:396-407, 2016.

[13] T. Jakubowski and K. Szczypkowski. Time-dependent gradient perturbations of fractional Laplacian. J. Evol. Equ., 10(2):319-339, 2010.

[14] N. Ju. The maximum principle and the global attractor for the dissipative 2D quasi-geostrophic equations. Comm. Math. Phys., 255(1):161-181, 2005.

[15] K. Kaleta and P. Sztonyk. Estimates of transition densities and their derivatives for jump Lévy processes. $J$. Math. Anal. Appl., 431(1):260-282, 2015.

[16] Y. Maekawa and H. Miura. Upper bounds for fundamental solutions to non-local diffusion equations with divergence free drift. J. Funct. Anal., 264(10):2245-2268, 2013.

[17] J. Mał ecki and G. Serafin. Hitting hyperbolic half-space. Demonstratio Math., 45(2):337-360, 2012.

[18] C. J. Niche and M. E. Schonbek. Decay of weak solutions to the 2D dissipative quasi-geostrophic equation. Comm. Math. Phys., 276(1):93-115, 2007.

[19] C. J. Niche and M. E. Schonbek. Decay characterization of solutions to dissipative equations. J. Lond. Math. Soc. (2), 91(2):573-595, 2015.

[20] J. Pedlosky. Geophysical Fluid Dynamics. Springer-Verlag New York, 1987.

[21] H. Rafeiro, N. Samko, and S. Samko. Morrey-Campanato spaces: an overview. In Operator theory, pseudodifferential equations, and mathematical physics, volume 228 of Oper. Theory Adv. Appl., pages 293-323. Birkhäuser/Springer Basel AG, Basel, 2013.

[22] S. G. Resnick. Dynamical problems in non-linear advective partial differential equations. PhD thesis, University of Chicago, 1995.

[23] P. Sztonyk. Regularity of harmonic functions for anisotropic fractional Laplacians. Math. Nachr., 283(2):289311, 2010.

[24] J. Wu. Dissipative quasi-geostrophic equations with $L^{p}$ data. Electron. J. Differential Equations, pages No. $56,13,2001$.

Faculty of Pure and Applied Mathematics, WrocŁaw University of Science and Technology, Wyb. Wyspiańskiego 27, 50-370 Wroceaw, Poland

E-mail address: tomasz.jakubowski@pwr.edu.pl

E-mail address: grzegorz.serafin@pwr.edu.pl 